\title{
Incorporation of realistic delivery limitations into dynamic MLC treatment delivery
}

\author{
Dale W. Litzenberg, ${ }^{\text {a) }}$ Jean M. Moran, and Benedick A. Fraass \\ Department of Radiation Oncology, University of Michigan Medical Center, Ann Arbor, \\ Michigan 48109-0010
}

(Received 28 September 2001; accepted for publication 22 February 2002; published 16 April 2002)

The clinical implementation of IMRT involves the use of a number of complex software-based systems, typically including an inverse planning system, a leaf sequencer, and a computercontrolled treatment delivery system. The inverse planning system determines the desired fluence patterns, the leaf sequencer translates those fluence maps into leaf trajectories, and the control system delivers those trajectories. While verification of intensity-modulated treatment fields has focused primarily on the dosimetric aspects of delivery, accurate delivery of the intended fluence distribution is dependent upon both the leaf sequencer and delivery control systems. Leaf sequencing algorithms typically do not incorporate many control system limitations, and this can lead to discrepancies between planned and delivered sequences. In this work, simple and complex fields were sequenced for the dynamic sliding window technique using different leaf speeds and tolerance settings to identify various limitations of the accelerator control system. This work was conducted on a Varian 2100 EX equipped with a Millennium 120 leaf MLC. The identified limitations were then incorporated into the sequencing algorithm using a limiting leaf velocity (less than the maximum leaf velocity), the leaf position tolerance, and the communications delay in the control system. Collision avoidance in leaf pairs was found to depend on a control system-enforced minimum gap between leaves and led to acceleration effects. By incorporating these effects into the leaf sequencing algorithm, dynamic sliding-window leaf sequences were produced which did not require beam interruptions or dose rate modulations for the parameter values used in calculating the sequence (dose rate, tolerance, leaf speed, and total monitor units). Incorporation of control system limitations into the leaf sequencing algorithm results in IMRT fields that are delivered with the prescribed constant dose rate, require less time to deliver, and have well-defined, calculable transmission dose characteristics. () 2002 American Association of Physicists in Medicine.

[DOI: $10.1118 / 1.1470499$ ]

Key words: DMLC, leaf sequencing, sliding window, system delay time, limiting velocity

\section{INTRODUCTION}

The advent of intensity modulated radiation therapy (IMRT) has required the development of new radiation delivery techniques to produce modulated fluence distributions. These distributions are typically generated by an inverse treatment planning system, and then transferred to a leaf sequencing algorithm ("leaf sequencer"). The sequencer is used to calculate the trajectories of pairs of MLC leaves to create the required fluence profiles. The dynamic multileaf collimator (DMLC) "sliding window" technique is one method used to deliver the modulated intensity distributions.

Using a multileaf collimator (MLC) to produce modulated patterns requires that leaves be used to block radiation. Because the leaves are not perfect attenuators, the transmission fluence must be accounted for when determining the leaf trajectories. Minimizing the transmission dose to surrounding healthy tissue may lead to fewer complications and a more conformal dose distribution. This constraint is typically achieved by minimizing the time (in MU) necessary to deliver the intensity distribution. ${ }^{1-3}$ To minimize delivery time, leaf sequencers typically call for one leaf of each pair to always move at its maximum velocity. While modifications to the basic algorithms, such as leaf synchronization, ${ }^{4}$ may slow down many of the leaves at any given time, at least one leaf is always moving at its maximum velocity.

While it is desirable to move the leaves as quickly as possible, it is also necessary that the leaves reach the calculated positions at the correct time, with minimal deviations from the prescribed trajectories. It has been shown that the dosimetric deviation to a given point is proportional to the error in the leaf gap. ${ }^{5}$ The gap size depends on several factors, especially the variation in the intensity distribution to be delivered under a given leaf pair and the dose rate. Smooth intensity distributions may have gaps of $5 \mathrm{~cm}$ or larger while distributions with large variations may, at times, require gaps of $1 \mathrm{~cm}$ or less. Thus, dose deviations of $2 \%$ would occur for gap errors of 0.1 to $0.02 \mathrm{~cm}$, respectively, for these gap sizes. Each leaf may have an allowable tolerance of \pm 0.05 to $\pm 0.01 \mathrm{~cm}$, respectively, in this scenario. In practice, however, it has been difficult to realize such tight tolerances. On a Varian MLC it is not possible to set a leaf tolerance smaller than $0.05 \mathrm{~cm}$, and at that value the number of beam holdoffs, due to tolerance violations, is unacceptable in most cases. By default, the tolerance value is quite often set to 0.1 
$\mathrm{cm}$, as is done in Varian's utility application (MLC Shaper) for viewing and creating sequences.

LoSasso $^{5}$ has proposed, based on careful dosimetric and delivery studies, that the tolerance be set to obtain the maximum reduction in treatment time with the smallest increase in tolerance. This technique is widely implemented by delivering a test sequence to determine either the delivery time, or number of beam hold-offs (which will be discussed in the next section), versus tolerance under clinical conditions. Typically, the delivery of IMRT sliding window sequences requires a tolerance of roughly $0.2 \mathrm{~cm}$ (at least when delivered with the Varian DMLC system), so that the field can be delivered without an unacceptable number of beam hold-offs or other interruptions in the delivery. The order of magnitude difference between the desired and achieved tolerances for these deliveries has never been explained.

In this article, we explain the origin of this discrepancy in terms of the limitations of the machine's control system and how this leads to beam hold-offs in the delivery. We then show how the limitations can be incorporated into the leaf sequencing algorithm to create dynamic sequences which will not require beam interruptions or dose rate modulations. The relative improvement, based on the reduction of the number of beam hold-offs, in delivering simple uniform fields and complex distributions is then demonstrated. The delivery and dosimetric implications for treatment planning and optimization are then discussed.

\section{MATERIALS AND METHODS}

Intensity-modulated fields used for tests were developed using the UMOpt inverse planning system. ${ }^{6-8}$ The test intensity patterns were transferred to the leaf sequencer, also developed in-house. The leaf sequencer has been modified from the algorithm described by Dirkx et al., and includes the effects of transmission and scatter. ${ }^{9}$ The effects of the rounded leaf-ends on the Varian MLC may be included as reported by Graves et al. ${ }^{10}$ Output from the sequencer includes the vendor-specific DMLC trajectory file which is required by the MLC control system.

The work presented here was performed with a Varian Clinac 2100EX accelerator equipped with a 120 leaf MLC (Varian Medical Systems, Palo Alto, Ca). This machine is equipped with 6 and $15 \mathrm{MV}$ photon beams and dynamic MLC capability ("DMLC") which allows dynamic motions of the MLC leaves while the beam is on.

The MLC and accelerator control system is schematically illustrated in Fig. 1. The MLC controller drives the MLC leaves linearly between control points that define the trajectories of the individual leaves. Through a communication link, the controller records the state of the accelerator (beam on or off), the dose fraction, the expected and actual leaf positions, and other information such as the user-selected tolerance. The acquisition, transfer, processing, and storage of this information requires a significant amount of time resulting in a roughly $0.055 \mathrm{~s}$ delay between the accelerator and MLC response to a given condition. The delay time inherent in this process is critical to the results described here.

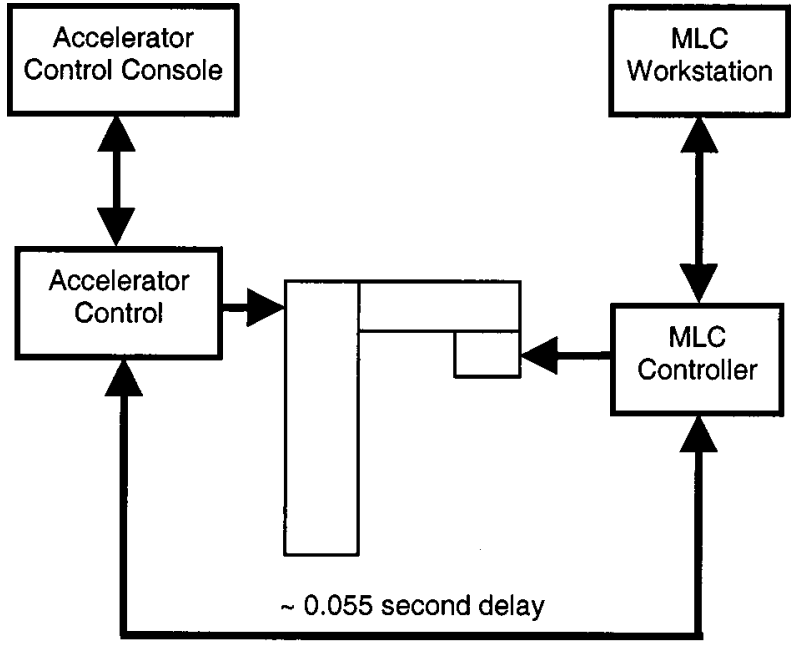

FIG. 1. Schematic of the control system depicting the communications link.

The MLC control system allows user-defined leaf position tolerance values ranging from 0.05 to $0.5 \mathrm{~cm}$. If one or more leaves are detected to be outside the tolerance envelope after each $0.055 \mathrm{~s}$ check cycle, then the beam is interrupted until all the leaves move within tolerance.

The time delay and the beam hold-offs cause several problems that need to be accounted for when calculating leaf sequences. As noted by others, ${ }^{11}$ when a hold-off is exerted by the MLC, the beam-on flag always remains set until the next check cycle, as shown in Fig. 2, indicating that dose is being delivered while at least one leaf is out of tolerance. In addition, data from the dynamic log files created from each delivery show that the actual leaf positions always lag their expected positions by at least one check cycle, as shown in Fig. 3. Finally, this delay has been noted to affect the dosimetric accuracy of step and shoot segmental delivery. ${ }^{12}$

To better understand the function of the control system, the delivery of a simple uniform intensity $10 \times 10 \mathrm{~cm}^{2}$ field (delivered dynamically) was evaluated, followed by further tests of more complex IMRT fields. A uniform intensity field of $100 \mathrm{MU}$ was chosen to avoid the complications that could potentially arise due to small gaps. The field was sequenced

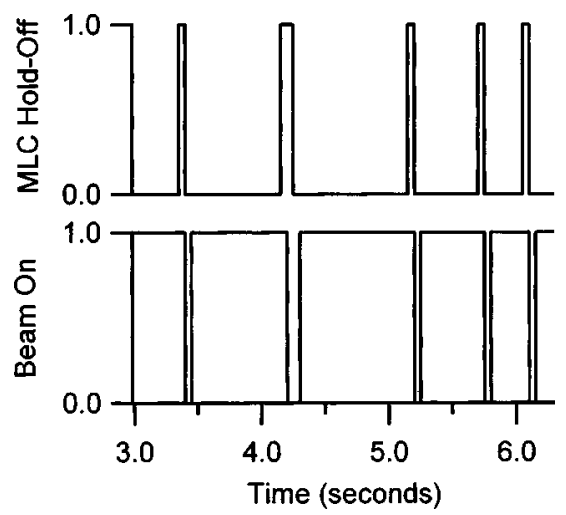

FIG. 2. Data from the dynamic sequence log files indicates that when a leaf is out of tolerance, the beam is not turned off ("beam on" flag $=0$ ) until one check cycle after the tolerance fault occurs ("MLC beam hold-off", $=0$ ). 


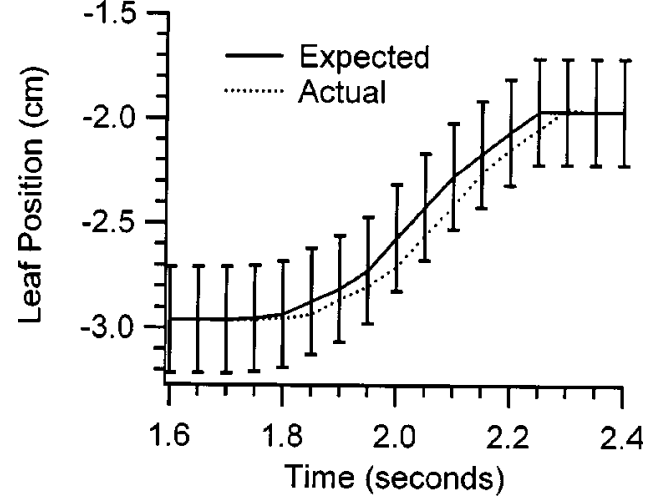

FIG. 3. Due to the inherent delay in the communications system between the MLC leaf motion controller and the accelerator the actual leaf position always lags its expected position by a time proportional to the delay time and the expected velocity. The error bars indicate the leaf position tolerance set by the user.

for multiple dose rates, tolerance values (from 0.05 to 0.5 $\mathrm{cm}$ ), and maximum leaf speeds of $1,2,3,4,5$ and $7 \mathrm{~cm} / \mathrm{s}$. (Sequences were calculated with maximum speeds exceeding the speed physically attainable by the leaves to study the effects of dose rate modulation and beam hold-offs.) The range of gap sizes for the sliding window ranged from 0.04 to $10 \mathrm{~cm}$ for all sequences. After each sequence was delivered, the logged dynamic sequence data were written to disk (files DynlogA.txt and DynlogB.txt) and analyzed using a program which compares the expected and actual leaf positions during the DMLC delivery. ${ }^{13,14}$

Using the series of tests described above, the effect of the tolerance value and maximum leaf velocity specified in the leaf sequencer were evaluated. These tests identified a number of important relationships between the leaf velocity, the leaf position tolerance, and time delay of the control system, all of which needed to be added to the sequencer to improve the deliverability of a sequence. The control system functionality was further tested for the special case where leaf pairs had a zero gap during part of the delivery. Finally, the collision avoidance limitations and acceleration affects were incorporated. When these effects are incorporated, there are no beam hold-offs, the dose rate is constant, and it is shown that the temporal and total MU efficiencies of a sequence are identical. It is then possible to choose sequencing parameters based on the treatment directive before calculating the leaf sequence.

\section{RESULTS}

\section{A. Limiting velocity ( $\left.\boldsymbol{V}_{\text {Limit }}\right)$}

Leaf sequencing algorithms usually require one leaf of a pair to be moving at the maximum physical velocity to minimize the treatment time and transmission dose. [The maximum leaf speed typically ranges from 1 to $2.5 \mathrm{~cm} / \mathrm{s}$ depending on the manufacturer ("Millennium MLC Specification," RAD 5609, September 2000, Varian Medical Systems). $\left.{ }^{9}\right]$ However, the communications delay, described earlier, implies that the maximum velocity used should not be the

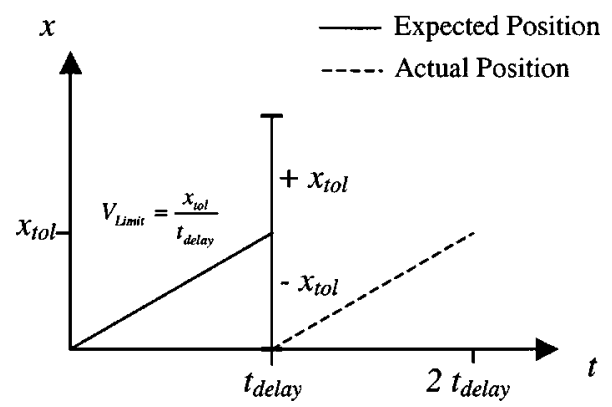

FIG. 4. The "limiting velocity" is determined by the acceptable tolerance (user-defined) and the delay time inherent in the communications system between the MLC leaf motion controller and the accelerator.

maximum speed of the leaves, but rather the largest velocity which will not put the leaves out of tolerance within the delay time of the communication system (as shown in Fig. 4). In other words, the maximum velocity should be replaced by the concept of the limiting velocity, $V_{\text {Limit }}$, which is given by

$$
V_{\text {Limit }}=\frac{x_{\text {tol }}}{t_{\text {delay }}},
$$

where $x_{\text {tol }}$ is the user-selected leaf position tolerance and $t_{\text {delay }}$ is the inherent time delay in the communications system. Equation 1(a) shows that there is a limiting minimum tolerance for a given leaf velocity. If the user selects a delivery tolerance below this limit, the leaf positions will always be out of tolerance after one clock cycle of the communications system and the beam will repeatedly shut off to allow the leaves to come into tolerance. Correspondingly, if the leaf sequencing algorithm uses a maximum velocity greater than the limiting velocity, the leaf will always be out of tolerance after one check cycle, as illustrated in Fig. 4. This relationship was tested using the delivery of the uniform field.

The measured number of MLC beam hold-offs versus tolerance for each sequence is shown in Fig. 5 for sequences

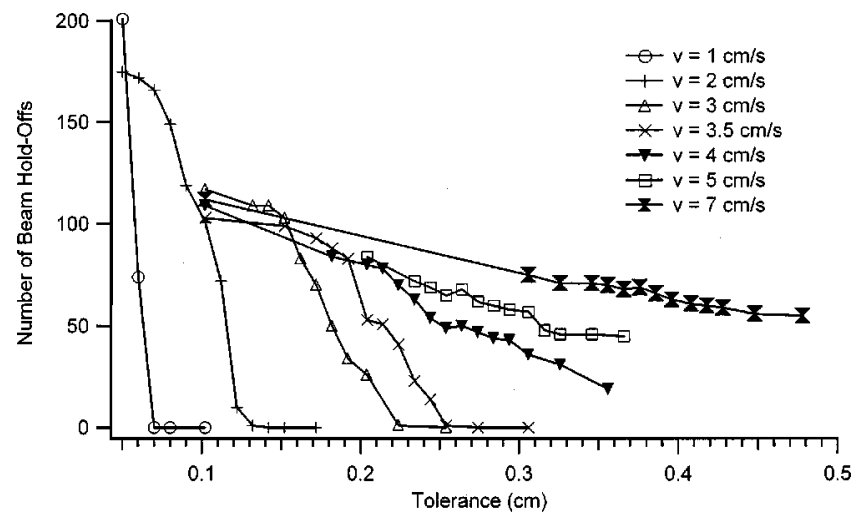

FIG. 5. The number of "MLC beam hold- offs" goes to zero as the tolerance increases beyond the limiting tolerance for the given leaf velocity. The width of the gradient indicates that there is significant variation in the period of the check cycle. As the velocity used to calculate the sequence exceeds the maximum physical velocity of the leaves, the shoulder vanishes over the allowable tolerance range. 
TABLE I. Summary of the calculated limiting tolerances, assuming a time delay of $0.055 \mathrm{~s}$, and the measured effective limiting tolerance, for leaf velocities between 1 and $3.5 \mathrm{~cm} / \mathrm{s}$.

\begin{tabular}{clcc}
\hline \hline Leaf velocity $(\mathrm{cm} / \mathrm{s})$ & $x_{\text {tol }}(\mathrm{cm})$ & $x_{\text {tol }}^{\text {eff }}(\mathrm{cm})$ & $100\left(x_{\text {tol }}^{\mathrm{eff}}-x_{\mathrm{tol}}\right) / x_{\mathrm{tol}}$ \\
\hline 1.0 & 0.055 & 0.07 & 27.3 \\
2.0 & 0.11 & 0.13 & 18.2 \\
3.0 & 0.165 & 0.23 & 39.4 \\
3.5 & 0.1925 & 0.26 & 35.1 \\
\hline \hline
\end{tabular}

generated using maximum leaf velocities ranging from 1 to 7 $\mathrm{cm} / \mathrm{s}$. Table I summarizes the calculated limiting tolerance from (1a), assuming a delay time of $0.055 \mathrm{~s}$, and the measured effective limiting tolerance where the number of beam hold- offs go to zero. The plateau region of the curves below the limiting tolerance indicates that the beam shuts off approximately every other clock cycle of the communications system when leaves are moving, to bring the leaves back within the set tolerance. The number of tolerance faults falls to zero for tolerances larger than the corresponding minimum tolerance since it becomes impossible for the leaves to move far enough at that speed to go out of tolerance in one clock cycle (given that there are no finite acceleration effects included in the leaf sequencer). The width of the shoulder in Fig. 5 indicates there is some variation in the communications delay. The tolerance value at which the number of MLC beam hold-offs goes to zero is about $30 \%$ to $40 \%$ larger than the calculated limiting tolerance due to this variation. This suggests that in practice the value used for the system delay time should be increased by a factor, $\alpha$, to account for the variation in the delay, where $\alpha$ in this case would be 1.4. This factor should be measured and set appropriately for each machine. The effective limiting velocity, which includes variations in the clock cycle of the control system, is then given by

$$
V_{\text {Limit }}^{\text {eff }}=\frac{x_{\text {tol }}}{\alpha t_{\text {delay }}} .
$$

This relationship assumes that the leaves have infinite acceleration, that the MLC controller does not impose limited acceleration, or move the leading leaf before the trailing leaf (to avoid collisions).

These experiments show that the increase in the number of hold-offs with decreasing tolerance values is due to the time delay between the accelerator and MLC control systems. The leaf motion controller anticipates the leaves moving out of tolerance if Eq. (1b) is not implemented. For situations where the tolerance value is set to a small value and the sequence is calculated for the maximum leaf speed, planned MLC beam hold-offs are used to allow the leaves to reach their expected location. When these sequences are run under such conditions the dose rate appears very unstable. This is quite often interpreted as system-optimized dose rate modulation to allow the leaves to move at their maximum velocity. Instead, the MLC is exerting a beam hold-off to allow the leaves to catch up to their expected locations. Dose rate modulation, in the intended sense, does not actually oc- cur unless the sequence is calculated using a velocity larger than $V_{\max }$. In cases where the effective limiting tolerance is larger than $0.5 \mathrm{~cm}$ (the largest selectable value) and the velocity used to calculate the sequence is greater than $V_{\max }$, the beam will be interrupted and the dose rate modulated. If the dose rate for each segment is reduced, or modulated, by a factor of $\left(V / V_{\max }\right)$ instead of $\left(V / V_{\text {Limit }}\right)$, the leaves will also be out of tolerance resulting in MLC beam hold-offs. Consequently, sequences that require dose rate modulation will be very demanding to deliver and may give unpredictable dosimetric results and should be avoided.

\section{B. Leaf pair collision avoidance}

When the leaves in a pair are very close to each other, acceleration rules must be imposed by the MLC controller to avoid collisions. It is important to distinguish this restricted acceleration from the classical quantity, $a_{x}=d^{2} x / d t^{2}$, which depends only on the leaf and motor characteristics. (This will be briefly discussed at the end of this section.) It is clear that when the gap between the leaves is zero, or very small, the leading leaf should move first or have a greater acceleration, to avoid a collision. Consequently, acceleration of the trailing leaf must be limited or delayed, potentially leading to larger gaps than prescribed, leaf position tolerance violations and beam hold-offs. The collision avoidance acceleration rules implemented by the manufacturer (including the size of the minimum gap) may depend on the position and velocity of the leading and trailing leaves, the acceleration of the leading leaf, and the time, $t_{\text {delay }}$, between leaf position verifications.

To evaluate the situation where leaves are closed and then opened again at some point during the delivery, complex intensity patterns were studied. One of the test fields was taken from a nonclinical head and neck case used for a treatment planning study. Seventy percent of the $1 \times 1 \mathrm{~cm}^{2}$ elements in this $8 \times 8 \mathrm{~cm}^{2}$ field were at or below the minimum transmission values of $1 \mathrm{MU}$. The field required $54 \mathrm{MU}$ to deliver and had a maximum modulation sum [second term in Eq. (3), to be discussed later] of $23 \mathrm{MU}$. This situation occurs at the beginning of most IMRT fields and for elements in the distribution where the prescribed fluence is less than the transmission fluence. The Varian MLC control system enforces a minimum gap of $0.05 \mathrm{~cm}$ for leaves that move at any time during a sequence as one rule of collision avoidance (private communication with Varian Medical Systems). To observe the behavior of leaves in this situation, we modified the leaf sequencer to include a minimum gap parameter, then sequenced intensity distributions that contained many elements with values below the minimum transmission value. The sequences were then delivered and the DynaLog files saved. We then examined the position versus time data from the Dynalog files for the involved leaves.

The data in the Dynalog files show that it is possible for the leaves to be less than $0.1 \mathrm{~cm}$ apart if they are moving at the same constant velocity, but below this minimum gap the controller imposes acceleration rules to avoid collisions when the trajectory changes. If the prescribed gap between leaf pairs is less than $0.10 \mathrm{~cm}$, the trailing leaf will not begin 


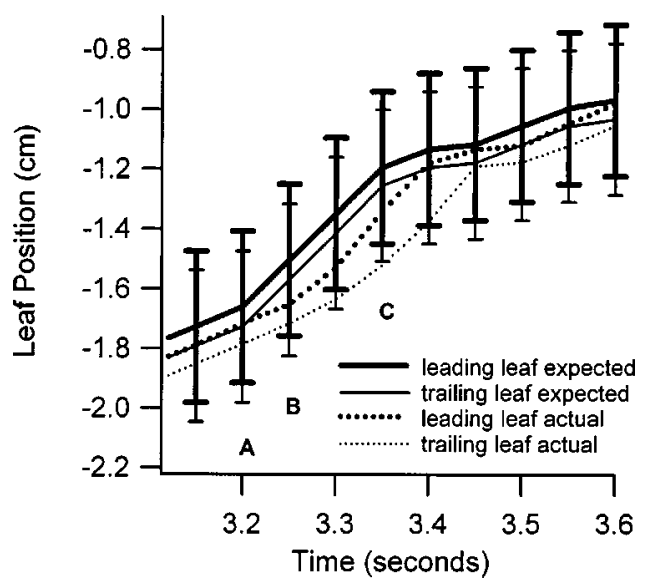

FIG. 6. The expected leaf trajectories (solid lines) show that both leaves are supposed to accelerate at time A. The actual recorded trajectories (dashed lines) show that the change in velocity does not occur until the next check cycle, time B. However, because the leaves are less than $0.1 \mathrm{~cm}$ apart $(0.07$ $\mathrm{cm}$ ) at time B the control system will not allow both of them to accelerate simultaneously. The leading leaf reaches the prescribed velocity two check cycles late while the trailing leaf takes four cycles. As a result, the trailing leaf is out of tolerance at time C, causing the MLC to exert a beam hold-off. (Error bars show the user-set tolerance.)

to accelerate until the check cycle after the leading leaf moves, roughly $0.055 \mathrm{~s}$ later, which results in a larger gap than expected, as shown in Fig. 6. In addition, the trailing leaf may not accelerate as quickly as prescribed under these conditions, again leading to a larger gap than prescribed, and potentially resulting in a tolerance violation. It therefore appears that the minimum allowable gap enforced by the MLC controller is not $0.05 \mathrm{~cm}$ but $0.1 \mathrm{~cm}$, or twice the minimum selectable leaf tolerance of $0.05 \mathrm{~cm}$ per leaf.

However, implementing the limiting velocity concept with a minimum gap of $0.1 \mathrm{~cm}$ does not eliminate leaf tolerance violations, nor, consequently, beam hold-offs, due to the small-gap acceleration rules imposed by the MLC controller. Small variations in the leaf velocities frequently result in smaller gaps than allowed $(0.1 \mathrm{~cm})$. When this happens, the acceleration rules imposed on the trailing leaf to avoid collisions may lead to a larger than desired gap, tolerance violations, and more beam hold-offs.

This behavior has led us to add the effects of leaf acceleration, for collision avoidance, into the leaf sequencing algorithm. Our current algorithm thus uses the following simple acceleration scheme: leaves that are not modulating the fluence are accelerated to the effective limiting velocity in four equally spaced velocity steps over a $4 \mathrm{~mm}$ distance. This scheme was chosen based on the typical acceleration scheme imposed on the trailing leaf, as implemented by the MLC controller when a small gap occurs. This, in combination with a $0.11 \mathrm{~cm}$ minimum gap, allowed the prescribed small gap to be maintained and eliminated beam hold-offs due to tolerance violations. It should be noted, while the scheme described above works, it may not be the optimal acceleration algorithm. By introducing acceleration, the average velocity is decreased. This will have very little effect on uniform fields but will increase the delivery time, and hence the transmission dose, slightly for nonuniform distributions. When the above acceleration mechanism is invoked, the time to move $1 \mathrm{~cm}$ is $43 \%$ longer than when the leaf moves at the constant limiting velocity (see the Appendix).

When the limiting velocity and an acceleration scheme are not implemented, positional overshoots may be quite frequent and greater than $0.1 \mathrm{~cm}$ depending on the parameters used for sequencing. These overshoots may produce tolerance violations, beam hold-offs, and often require two or three check cycles to move the leaf back to its prescribed position. A scheme for slowing down the leaves, to avoid overshoot, is not necessary to eliminate beam hold-offs when the concept of the limiting velocity and an acceleration scheme are implemented. Data from the Dynalog files indicates that overshoots still occur occasionally. However, due to the time delay in the control system, the leaves always lag their prescribed positions and overshoots have only been observed in instances where leaves are expected to stop. In these cases the greatest overshoot observed is $0.0001 \mathrm{~cm}$. When an overshoot occurs, the leaf moves backwards to the prescribed position by the following check cycle. Consequently, because the leaf is stopped, and within tolerance, no beam hold-offs occur.

Implementing the acceleration scheme described above restricts the acceleration of the leaves to be less than the physical limit determined by the characteristics of the leaf and motor assembly. Consequently, it is not necessary to account for the finite physical acceleration of a leaf to eliminate tolerance violations and beam hold-offs.

\section{Segmenting leaf trajectories}

After the leaf trajectories have been calculated, they are segmented by interpolation. A sufficient number of segments should be used to preserve the acceleration characteristics included in the calculation of the leaf trajectories. The appropriate fluence per segment is determined by the dose rate and the time scale of the acceleration scheme. However, the step should not be smaller than that determined by the dose rate and the delay time in the communications system. Therefore the number of uniform segments is

$$
N_{\mathrm{seg}}=\frac{T_{\mathrm{MU}}}{\Delta T_{\mathrm{MU}}},
$$

where

$$
\Delta T_{\mathrm{MU}} \cong \max \left\{\begin{array}{l}
R\left[\frac{4}{V_{\text {Limit }}}\right](0.1 \mathrm{~cm}), \\
R t_{\text {delay. }}
\end{array}\right.
$$

When the number of segments is determined this way it is possible that the number may be quite large is some cases. In these cases, the segmentation scheme described by Dirkx et $a l .{ }^{9}$ would reduce the number of segments. In this technique, the segments are ranked, highest to lowest, by the change in the leaf velocity and the desired number of segments kept.

Dividing the leaf trajectories into segments defines a minimum dose increment. In the same way, some segment- 
ing algorithms ${ }^{9}$ may round leaf positions to the nearest position interval, determined perhaps by the resolution of the fluence grid. If the leaves are not capable of traveling the distance increment, $\Delta x$, in the time taken to deliver the dose increment, $R N_{\mathrm{seg}} / T_{\mathrm{MU}}$, the leaves will not be able to reach their prescribed positions and beam hold-offs will occur. If both quantities are discretized, the number of segments should be chosen so that the effective limiting velocity is not exceeded:

$$
\begin{aligned}
& \Delta x\left[\frac{R}{\left(T_{\mathrm{MU}} / N_{\mathrm{seg}}\right)}\right] \leqslant V_{\text {Limit }}, \\
& N_{\mathrm{seg}} \leqslant \frac{V_{\mathrm{Limit}} T_{\mathrm{MU}}}{\Delta x R} .
\end{aligned}
$$

This limits the number of segments allowable for reliable delivery and may prevent adequate sampling of the leaf trajectory, especially in the case where uniform dose increments are used. For this reason, discretization of the leaf position should be avoided.

\section{Incorporation of control system limits into leaf sequencing algorithm}

In implementing Eq. (1b) it should be noted that the effective limiting velocity can never exceed the maximum physical velocity of the leaf without inducing dose modulation which slows down delivery and causes tolerance faults. The equation should instead be used to find the minimum tolerance setting for the highest reliable velocity. For example, if the user sets the tolerance to $0.5 \mathrm{~cm}$ to obtain the quickest delivery, the effective limiting velocity would be 6.5 $\mathrm{cm} / \mathrm{s}$, which is well above the maximum leaf speed of most MLCs (assuming $\alpha=1.4$ and $t_{\text {delay }}=0.055 \mathrm{~s}$ ). On the other hand, if the maximum reliable velocity is $2.5 \mathrm{~cm} / \mathrm{s}$ and this value is used to calculate the leaf sequence, then leaf position tolerance faults will not occur when the tolerance is set above $0.2 \mathrm{~cm}$, as others ${ }^{5}$ have noted.

When the concept of the limiting velocity is implemented and the minimum gap and leaf acceleration effects (for collision avoidance) are incorporated into the leaf sequencing algorithm, it is possible to produce dynamic "sliding window" sequences that can be delivered at a constant dose rate with no MLC induced beam hold-offs. This requires that the sequence be delivered at the prescribed dose rate, tolerance setting, and calculated MU value. The relative improvement in delivery, based on hold-offs, is shown in Fig. 7. The total number of $0.055 \mathrm{~s}$ check cycles required to deliver the uniform and complex sequences are shown along with the number of cycles where beam hold-offs were exerted. Sequences generated with the "standard" method were delivered at the prescribed dose rate of $400 \mathrm{MU} / \mathrm{min}$, the calculated number of $\mathrm{MU}$, the default tolerance setting of $0.1 \mathrm{~cm}$ and sequenced with a leaf velocity of $3.0 \mathrm{~cm} / \mathrm{s}$. When only the limiting velocity was implemented, there was a substantial reduction in the number of beam hold-offs, especially for the more complex cases. When the minimum gap and leaf acceleration were also included, all beam hold-offs were avoided, al-

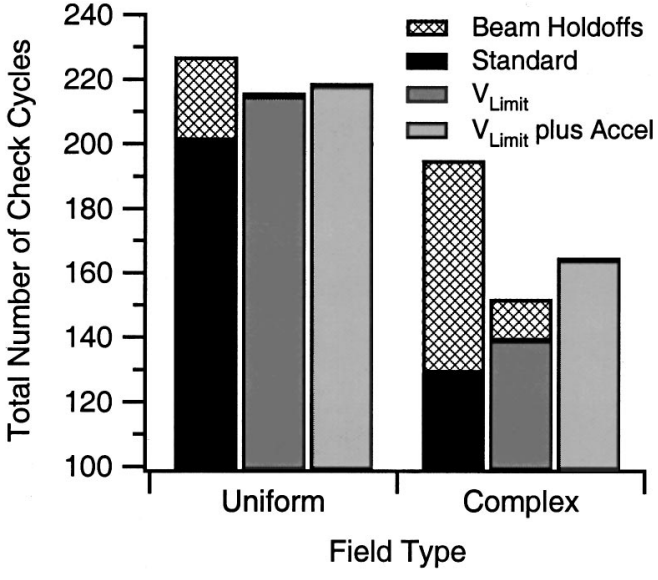

FIG. 7. This figure illustrates the number of check cycles and beam hold-offs required to deliver a simple $10 \times 10 \mathrm{~cm}^{2}$ uniform field and a sample complex clinical field, when different effects are included in the "sliding window" sequencing algorithm. There is a significant reduction in beam hold-offs when the limiting velocity concept is implemented. In both cases, all holdoffs are eliminated when limiting velocity, minimum gap and acceleration effects are included.

though it took slightly longer to deliver the sequence $(0.478$ seconds/centimeter of field width when the acceleration scheme is implemented with, $V_{\text {Limit }}^{\text {eff }}=3.0 \mathrm{~cm} / \mathrm{s}$ ).

The maximum effective limiting velocity should not be set higher than the manufacturer's specified maximum possible leaf speed. The data in Fig. 5 indicates that the involved leaves had a maximum leaf speed between 3.5 and $4.0 \mathrm{~cm} / \mathrm{s}$. This data was obtained using the central 20 leaf pairs on a Varian Millennium 120 leaf collimator which project to 0.5 $\mathrm{cm}$ wide at isocenter. However, the first and last ten leaf pairs are twice as thick and move noticeably slower. The manufacturers maximum leaf speed specification is $2.5 \mathrm{~cm} / \mathrm{s}$, presumably set below the true maximum physical speed of the slowest leaves to ensure reliable performance.

\section{ANALYSIS}

The inherent delay in the communications between the accelerator and the MLC leaf motion controller has several implications for the delivery of intensity-modulated fluence patterns. The first consequence of Eq. (1b) is that there is a trade-off between leaf position accuracy and leaf speed. It is not possible to set small tolerances and use fast leaf speeds without causing beam hold-offs due to the inherent system delay. The second implication is that if the effective limiting velocity is used to calculate the leaf sequence, the leaves will never go out of tolerance, provided the MLC is working properly, and the dose rate will remain constant for the duration of the sequence. Another consequence is that the first segment of a dynamic delivery will be overdosed by an amount $\left(R t_{\text {delay }}\right)$, where $R$ is the dose rate, while the last segment will be underdosed by the same amount, $\left(R t_{\text {delay }}\right)$. This is because the accelerator turns on before the leaves start moving, by an amount of time determined by the delay in the communication system, and turns off early by the same amount of time. This, however, may be corrected, by 
adjusting the desired dose in the first and last segments by the appropriate amount. The appropriate tradeoff between leaf speed and leaf position accuracy is more difficult to determine and will depend on other factors such as dose rate. Understanding the impact of these parameters and choosing them appropriately will help achieve the treatment directive by minimizing the treatment time or the transmission dose.

To address the dependence of these factors on each other, the delivery of a sequence is evaluated in both MU and time. The total treatment time in MU may be determined by applying Eq. (5) in Spirou et al. ${ }^{1}$ to each leaf pair to find the pair that will take the longest time to finish. The equation is recast here to express the effective limiting velocity, $V_{\text {Limit }}^{\text {eff }}$, in units of $\mathrm{cm} / \mathrm{s}$. The total treatment time, $T_{\mathrm{MU}}$, in units of $\mathrm{MU}$ is given by

$$
\begin{aligned}
T_{\mathrm{MU}}= & (1-\tau)\left(x_{N}-x_{m}\right) \frac{R}{60 V_{\mathrm{Limit}}^{\mathrm{eff}}}+F\left(x_{m}\right) \\
& +\sum_{i=m}^{N-1}\left[F\left(x_{i+1}\right)-F\left(x_{i}\right)\right]_{F\left(x_{i+1}\right)>F\left(x_{i}\right)},
\end{aligned}
$$

where the dose rate, $R$, is in MU/min, $x_{N}$ is the ending leaf position, $x_{m}$ is the position where the slope of the fluence profile first becomes negative (both are in units of $\mathrm{cm}$ ), the fluence values, $F$, are in units of $\mathrm{MU}\left[F\left(x_{0}\right)=0\right]$, the limiting velocity, $V_{\text {Limit }}^{\text {eff }}$, is in $\mathrm{cm} / \mathrm{s}$ and the transmission factor, $\tau$, is a dimensionless number between 0 and 1 . If leaf synchronization is included in the algorithm to remove tongueand-groove effect, all leaves are required to start at the same left-most position, making $\left(x_{N}-x_{m}\right)=w$, the total width of the field. If the transmission term is also separated out, (2) becomes

$$
\begin{aligned}
T_{\mathrm{MU}}= & \frac{R w}{60 V_{\mathrm{Limit}}^{\mathrm{eff}}}+\sum_{i=0}^{N-1}\left[F\left(x_{i+1}\right)-F\left(x_{i}\right)\right]_{F\left(x_{i+1}\right)>F\left(x_{i}\right)} \\
& -\frac{R w}{60 V_{\text {Limit }}^{\mathrm{eff}}} \tau .
\end{aligned}
$$

The first two terms give the time in MU it would take to deliver the sequence if there were no transmission. The first term gives the minimum time, in MU, that it would take for the leaves to cross the field at their effective limiting velocity. The second term accounts for the additional time needed for the leaves to modulate the intensity. The last term indicates that the delivery time is shortened due to transmission through the MLC leaves. Due to the conformal nature of IMRT, most fields have many fluence elements over normal healthy tissue that, optimally, would receive zero dose, indicating that the third term should be minimized if possible.

When sequences are created using the effective limiting velocity, the sequence can proceed to completion at the dose rate used to calculate the sequence. This is true as long as $V_{\text {Limit }}^{\text {eff }} \leqslant V_{\text {max }}$ and the programmed dose rate on the accelerator at the time of delivery is the same dose rate used to calculate the sequence. Because the dose rate is then constant, the total time, in seconds, to deliver the sequence is

$$
\begin{aligned}
T_{\text {time }}= & \frac{T_{\mathrm{MU}}}{R}, \\
T_{\text {time }}= & (1-\tau) \frac{w}{V_{\text {limit }}^{\text {eff }}}+\frac{60}{R} \sum_{i=0}^{N-1}\left[F\left(x_{i+1}\right)\right. \\
& \left.-F\left(x_{i}\right)\right]_{F\left(x_{i+1}\right)>F\left(x_{i}\right)}, \\
T_{\text {time }}= & (1-\tau) \frac{w \alpha t_{\text {delay }}}{x_{\text {tol }}}+\frac{60}{R} \sum_{i=0}^{N-1}\left[F\left(x_{i+1}\right)\right. \\
& \left.-F\left(x_{i}\right)\right]_{F\left(x_{i+1}\right)>F\left(x_{i}\right)} .
\end{aligned}
$$

As mentioned, ${ }^{1}$ forcing the leaf sequencer to minimize the beam-on time in MU does not minimize the time of the treatment. This is due to the second term in (8) that accounts for the time taken to modulate the fluence across the field. Using typical values of $w=10.0 \mathrm{~cm}, t_{\text {delay }}=0.055 \mathrm{~s}, x_{\text {tol }}$ $=0.1 \mathrm{~cm}$ and $\tau=0.02$, it can be seen that the first term is rather small and that the second term (the modulation term) will typically dominate in determining the temporal time to deliver the sequence.

The amount of transmission at any point in the field is given by the product of the transmission factor and the amount of time, in MU, that the point was covered by a leaf. The maximum transmission will therefore occur in regions where zero fluence is prescribed. (If transmission has been included in the leaf sequencing algorithm, elements whose prescribed value is greater than or equal to the transmission value should receive the prescribed fluence.) The transmission fluence to areas of zero prescribed fluence is found by multiplying (7) by the transmission factor giving

$$
F_{\text {trans }}^{\max }=\tau T_{\mathrm{MU}} \text {. }
$$

Thus the transmission dose to normal healthy tissue is minimized in the same way that the length of the treatment, in MU, is minimized.

Figures $8(a)-8(d)$ illustrate the behavior of these equations and the relative importance of each term. The total number of MU and the total time required to deliver profiles are shown as a function of the effective limiting tolerance. These figures show two examples where the sum in the modulation term is $100 \mathrm{MU}$ [Figs. 8(a) and 8(b)] and 600 MU [Fig. 8(c) and 8(d)]. Total treatment times in MU and in seconds are calculated at dose rates between 100 and 600 $\mathrm{MU} / \mathrm{min}$ and for tolerances between 0.02 and $0.5 \mathrm{~cm}$. Typical values of field width, $w=10 \mathrm{~cm}$, system delay, $t_{\text {delay }}$ $=0.055 \mathrm{~s}$, and transmission, $\tau=0.02$, have been used for these examples. (Note that when $t_{\text {delay }}=0.055 \mathrm{~s}$ and $V_{\max }$ $=3.0 \mathrm{~cm} / \mathrm{s}$, the effective limiting tolerance is $0.23 \mathrm{~cm}$. Reductions in MU and time are not possible for higher tolerances unless the maximum physical leaf speed is increased by the manufacturer.)

The maximum transmission fluence to normal healthy tissue, as given by Eq. (9), is shown on the right axes of Figs. $8(\mathrm{a})$ and $8(\mathrm{c})$. The most important factor in reducing the total transmission dose is minimizing the modulation term in Eq. (3). Fields that are uniform or slowly varying require the 

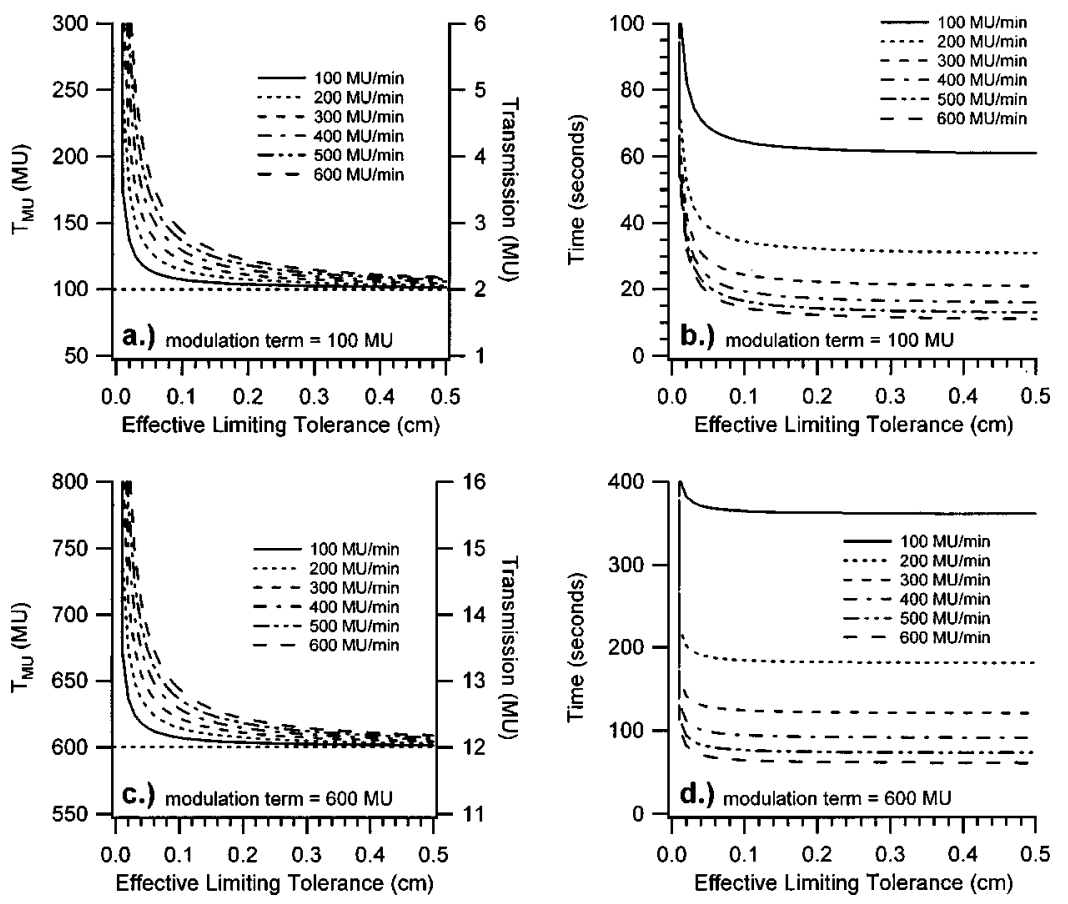

FIG. 8. Minimizing the treatment time in MU does not minimize the treatment time temporally and both quantities vary with the effective limiting tolerance, dose rate and field width. Examples are shown of two hypothetical fluence profiles whose fluence modulation term sums to $100 \mathrm{MU}$ and $600 \mathrm{MU}$. (a) shows the transmission fluence and the total MU required to deliver a field whose modulation term sums to $100 \mathrm{MU}$ as a function of effective limiting tolerance and at different dose rates while (b) shows the corresponding treatment time in seconds. (c) shows the transmission fluence and the total MU required to deliver a field whose modulation term sums to $600 \mathrm{MU}$ as a function of effective limiting tolerance and at different dose rates while (d) shows the corresponding treatment time in seconds. fewest MU to deliver and result in the least transmission. Various combinations of dose rate and tolerance setting (effective limiting velocity) may further increase the transmission fluence by an additional $1 \mathrm{MU}$ for tolerances of $0.1 \mathrm{~cm}$ or greater.

In general, the total treatment time, temporally and in total dose, is decreased by using the highest acceptable effective limiting velocity (or tolerance) and by minimizing the modulation term. Slowly varying, smooth fluence profiles require less time and fewer MU to deliver than profiles with large variations from element to element. Further reductions may be achieved by minimizing the field width. This could be achieved by including a strong penalty in the treatment planning optimization engine against perimeter elements whose values are less than that due to transmission. As shown in Fig. 8, increases in dose rate may reduce the delivery time by as much as a factor of 5 with a relatively modest increase (5\% to $40 \%$ ) in the number of MU required to deliver the sequence, for tolerances larger than $0.1 \mathrm{~cm}$. When the tolerance is increased (or the limiting velocity decreased), the delivery time does not change significantly $(\sim 5 \%)$ while the total MU required slightly decreases $(5 \%-$ $40 \%$ ), leading to more efficient delivery. Careful consideration must be given to using effective limiting tolerances below $0.1 \mathrm{~cm}$. In these cases the treatment directive must be considered to select the proper dose rate and tolerance.

\section{A. Delivery efficiency}

All of the tradeoffs may be summarized by examining the efficiency of the sequence. If Eqs. (7) and (8b) are divided by the modulation term, we get a fraction expressing the efficiency of the sequence. This operation gives the same result for Eqs. (7) and (8b), indicating that the dose and temporal efficiencies are the same when the dose rate is constant:

$$
E=\frac{(1-\tau) R w}{60 V_{\text {Limit }}^{\text {eff }} \Sigma_{l=0}^{N-1}\left[F\left(x_{i+1}\right)-F\left(x_{i}\right)\right]_{F\left(x_{i+1}\right)>F\left(x_{i}\right)}}+1
$$

Substituting Eq. (7) into Eq. (9) and dividing by the modulation term gives the cost in transmission dose to normal healthy tissue for inefficient treatment delivery beyond the ideal value for the desired fluence pattern.

$$
C_{\text {trans }}^{\max }=\tau E \text {. }
$$

Ideally the efficiency would be 1 if there were no leaf speed or transmission effects. Values between 1 and 2 indicate that the modulation term is dominant, while values larger than 2 indicate that the delivery will be dominated by leaf speed and transmission effects. As the efficiency value increases beyond a value of 2 , the cost in transmission dose to healthy tissue rises rapidly.

The sequence efficiency and cost in transmission dose are shown in Fig. 9 where typical values of field width, $w$ $=10 \mathrm{~cm}$, effective limiting velocity, $V_{\text {Limit }}^{\text {eff }}=2.5 \mathrm{~cm} / \mathrm{s}$, and transmission, $\tau=0.02$, were used. Curves are shown for dose rates between 100 and $600 \mathrm{MU} / \mathrm{min}$ for sequence modulations between 10 and $1000 \mathrm{MU}$. The temporal efficiency and dose efficiency of the sequence are minimized at the lowest dose rate and largest effective limiting velocity which, unfortunately, results in the longest delivery time and requires a large tolerance setting. Larger dose rates lead to less efficient sequences and increase the transmission dose but dramatically reduce the time required to deliver the sequence.

\section{B. Leaf position tolerances}

Finally, the effect of the user-defined leaf position tolerance on dosimetric accuracy needs to be addressed in the 


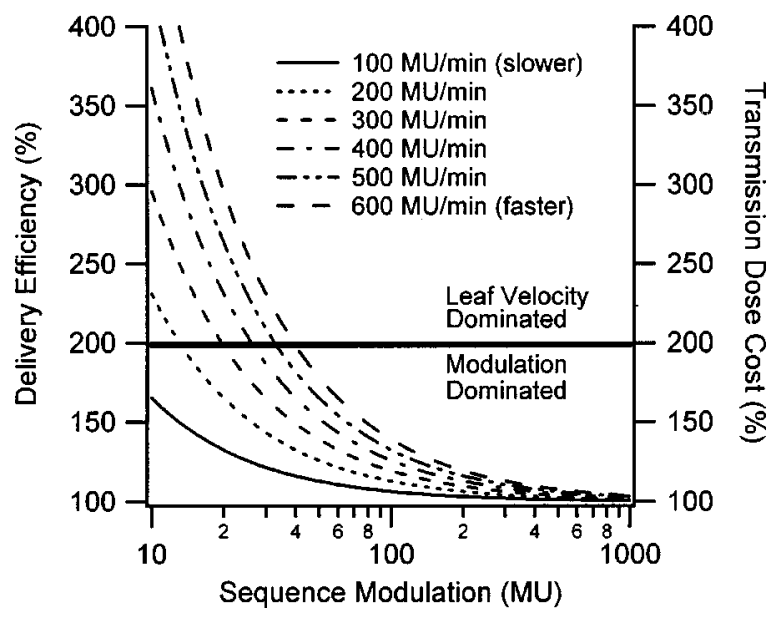

FIG. 9. The relationship between delivery efficiency and transmission dose cost versus sequence modulation.

context of the delivery system. The actual leaf position always lags the expected position due to the communications delay in the control system between the MLC leaf motion controller and the accelerator (Figs. 3 and 6). The MLC controller appears to make no attempt to correct for this (by moving the leaves faster than the requested velocity during that segment or subsequent segments). Consequently, the leaves are always behind the expected position by an amount determined by the system delay and the programmed effective limiting velocity, $\left(t_{\text {delay }} \times V_{\text {Limit }}^{\text {eff }}\right)$. This implies that while the first and last segments have dosimetric shifts, the intermediate segments will have temporal shifts in reaching their expected locations. However, because the dose rate is constant when using the effective limiting velocity, the segments will receive the prescribed amount of fluence. Consequently, if the first and last segments are corrected, the entire sequence should be dosimetrically and spatially accurate regardless of the dose rate or the leaf tolerance used to calculate the sequence.

A perturbation to the above argument may occur if a velocity dependent positional lag occurs during the check cycles that each leaf crosses the point of interest. In this case, dose variations as large as $R t_{\text {delay }}$ could be observed though they are likely to be a fraction of this value. This is particularly true with the acceleration scheme implemented. Because the acceleration scheme for the leading leaf is fairly well matched to the acceleration imposed on the trailing leaf by the MLC controller, the difference in lag times is very small.

These assertions are supported by previously reported data. ${ }^{5,11}$ In these cases, standard treatment fields used to treat prostate cancer were verified dosimetrically, using an ion chamber and film, over the range of tolerances from 0.05 to $0.5 \mathrm{~cm}$. In both cases, the delivered dose decreases to a stable minimum for tolerances greater than $0.2 \mathrm{~cm}$. It was also concluded $^{5}$ from tests that the dose variation below the 0.2 $\mathrm{cm}$ tolerance was due to beam instability and not variations in gap, which was shown could be maintained to better than $0.01 \mathrm{~cm}$. Consequently, it has been shown that when set ap- propriately, the leaf tolerance setting has little impact on dosimetric deliver of sequences and, as suggested by LoSasso, ${ }^{5}$ primarily serves as a safety interlock in the event that a leaf malfunctions during delivery.

\section{DISCUSSION}

Leaf sequencing algorithms have been the subject of a great deal of work over the last few years. ${ }^{1-5,9,11-14}$ However, few have considered in detail the effects that control system operations and limitations may have on the machine's ability to actually delivery the planned DMLC sequences. Recently, investigators have presented descriptions of some of these effects or limitations. ${ }^{12}$ However, the connection between these features and the control system has not always been elucidated. In this work, we describe in detail how the timing considerations for the Varian DMLC control system affect the DMLC sequences, and how to calculate stable leaf sequences for a given intensity pattern.

Leaf sequencing algorithms should incorporate the functionality of the delivery system to improve the accuracy and efficiency of IMRT delivery. At this time, many inverse planning/optimization systems do not incorporate delivery limitations when deriving desired intensity maps. As optimization systems continue to evolve, incorporation of realistic limitations (such as the transmission dose to critical structures) can be used to determine treatment plans that are optimized for delivery based on the treatment directive.

Because the leaf speed and dose rate are parameters used to calculate the leaf trajectories, they must be chosen prior to the calculation. The algorithm in turn calculates the number of MU to be programmed into the accelerator control console at the time of delivery. Sequences should never be delivered at a different dose rate than that used to calculate the sequence, nor should the total number of MU programmed on the console be different than that determined by the algorithm. Because leaf motion control systems prescribe leaf position as a function of dose fraction instead of absolute dose, using the wrong number of MU, or the wrong dose rate, can lead to severe mechanical delivery issues. In addition, using a different dose rate will change the transmission dose, potentially affecting patient outcome. For these reasons, utility programs for creating and reading dynamic sequences that do not display or provide control over these parameters may produce sequences with very unexpected delivery and dosimetric consequences and should only be used to examine leaf sequences created by a well benchmarked algorithm. For the same reasons, verification measurements of sequences should always be made using the prescribed dose rate and the calculated number of MU. If different values are used, the verification measurements may not be an adequate representation of the delivery during patient treatments.

Although the lowest transmission dose is obtained by choosing a low dose rate, there are situations where higher transmission may be acceptable if a higher dose rate will allow a sequence to be delivered more quickly and without interruption. For situations where IMRT is used to treat volumes that have significant intratreatment motion, the fastest 
treatment time may be desired to allow patient treatment under breath-hold. This is especially true in scenarios where patients are only capable of holding their breath for 10 to 20 s. ${ }^{15}$ While it is possible to interrupt a dynamic sequence to split delivery of the field over two or more breath holds, ideally it is better if the sequence can be delivered in one breath hold. This would result in shorter treatment times, less patient fatigue, less opportunity for positioning errors, better dosimetric delivery, and fewer chances for random delivery errors.

The length of time to deliver a sequence can also be shortened by changes in the control system. If the time delay can be shortened, it would (1) permit higher effective limiting velocities without increasing the leaf position tolerance, (2) have a significant impact on wide fields with low fluences, (3) permit higher effective limiting velocities, leading to less transmission dose, and (4) reduce the overdose which occurs in the first segment and the underdose which occurs in the last segment. Transmission dose could also be minimized by modifying the sliding window technique to have the jaws move dynamically, following the motion of the open leaf pairs. Of course, a design change of this magnitude has many other implications for the machine control system design and is not a trivial undertaking.

\section{CONCLUSIONS}

The ability of a DMLC control system to deliver a given dynamic sequence is affected by the real time behavior of the machine control system and particularly by the delay between the accelerator beam control and the DMLC leaf motion controls which occur in the Varian DMLC control system. If this delay time is not accounted for, DMLC delivery sequences are often forced to have larger-than-desired tolerances, and also experience numerous beam hold-offs, with even SMLC (segmental or step-and-shoot) deliveries being affected (overdose in the first segment and underdose in the last).

By defining a limiting velocity for the leaves, rather than the maximum velocity, it is possible to modify the leaf sequencing algorithm so that the effects of this time delay do not reduce the precision of the DMLC control, nor result in undesired beam hold-offs. We have shown that even simple fields (uniform intensity $10 \times 10 \mathrm{~cm}^{2}$ field) delivered with the DMLC technique can manifest many of these problems, and that the problems are resolved with use of the modified motion equations which are described here.

\section{ACKNOWLEDGMENTS}

We would like to thank Dr. Maarten Dirkx for providing the software and other assistance that was used in the development of the leaf sequencer reported here. Many people at Varian Medical Systems were helpful in understanding the control system behavior including Chris Lutz, Wayne Keranen, and Cal Huntzinger. Communication with Varian was greatly facilitated by Dr. Dan McShan and Dr. Marc Kessler. Support for this work was provided in part by NIH Grant No. P01-CA59827.

\section{APPENDIX: INCREASE IN TREATMENT TIME DUE TO LEAF ACCELERATION}

When an acceleration scheme is introduced, the average velocity of the leaves decreases. The scheme described is implemented on a $0.1 \mathrm{~cm}$ fluence grid. In the worst case, the scheme would be implemented in at least one of the leaf pairs for every centimeter of field width. When the leaves travel at the constant limiting velocity, the time taken to move $1 \mathrm{~cm}$ is

$$
t_{\mathrm{VLim}}=\frac{1.0}{V_{\text {Limit }}} \mathrm{s},
$$

where $V_{\text {Limit }}$ is in units of $\mathrm{cm} / \mathrm{s}$. However, when the leaves are accelerated to the effective limiting velocity in four equally spaced velocity steps over a $4 \mathrm{~mm}$ distance the time taken to travel $1 \mathrm{~cm}$ is

$$
\begin{aligned}
& t_{\text {accel }}=\frac{0.1 \mathrm{~cm}}{V_{\text {Limit }} / 4}+\frac{0.1 \mathrm{~cm}}{V_{\text {Limit }} / 2}+\frac{0.1 \mathrm{~cm}}{3 V_{\text {Limit }} / 4}+\frac{0.7 \mathrm{~cm}}{V_{\text {Limit }}}, \\
& t_{\text {accel }}=\frac{1.433}{V_{\text {Limit }}} \mathrm{s} .
\end{aligned}
$$

Thus, in the worst case, distributions sequenced with this acceleration scheme would require $43 \%$ more time to deliver. For a Varian MLC with a maximum leaf velocity of $2.5 \mathrm{~cm} / \mathrm{s}$, $t_{\text {accel }}=0.573 \mathrm{~s}$ per centimeter of field width.

${ }^{a)}$ Electronic mail: litzen@umich.edu

${ }^{1}$ S. Spirou and C. S. Chui, "Generation of arbitrary intensity profiles by dynamic jaws or multileaf collimators," Med. Phys. 21, 1031-1042 (1994).

${ }^{2}$ J. Stein, T. Bortfeld, B. Dorschel, and W. Schlegel, "Dynamic x-ray compensation for conformal radiotherapy by means of multileaf collimation," Radiother. Oncol. 32, 163-173 (1994).

${ }^{3}$ R. Svensson, P. Kallman, and A. Brahme, "Analytical solution for the dynamic control of multileaf collimators," Phys. Med. Biol. 39, 37-61 (1994).

${ }^{4}$ J. P. C. van Santvoort and B. J. M. Heijmen, "Dynamic leaf collimation without 'tongue and groove effects,'” Phys. Med. Biol. 41, 2091-2105 (1996).

${ }^{5}$ T. LoSasso, C. S. Chui, and C. C. Lang, "Physical and dosimetric aspects of a multileaf collimation system used in the dynamic mode for implementing intensity modulated radiotherapy," Med. Phys. 25, 1919-1927 (1998).

${ }^{6}$ M. L. Kessler, J. H. Kim, D. L. McShan, and B. A. Fraass, "A flexible and robust scoring methodolgy for automated optimization of conformal radiotherpy treatment plans," Int. J. Radiat. Oncol. Biol. Phys. (Suppl.) 45, 187-188 (1999).

${ }^{7}$ K. A. Vineberg, A. Eisbruch, M. M. Coselmon, D. L. McShan, M. L. Kessler, and B. A. Fraass, "Is uniform target dose possible in IMRT plans for the head and neck?," Int. J. Radiat. Oncol., Biol., Phys. (accepted).

${ }^{8}$ J. J. H. Kim, N. Dogan, D. L. McShan, and M. L. Kessler, "An AVSbased system for optimization of conformal radiotherapy treatment plans," in Proceedings of the 1995 International Advanced Visual Systems User and Developer Conference, Boston, MA (1995), pp. 417-423.

${ }^{9}$ M. L. P. Dirkx, B. J. M. Heijmen, and J. P. C van Santvoort, "Leaf trajectory calculation for dynamic multileaf collimation to realize optimized fluence profiles," Phys. Med. Biol. 43, 1171-1184 (1998).

${ }^{10}$ M. N. Graves, A. V. Thompson, M. K. Martel, D. L. McShan, and B. A. Fraass, "Calibration and quality assurance for rounded leaf-end MLC systems," Med. Phys. 28, 2227-2233 (2001). 
${ }^{11}$ C. Burman, C. S. Chui, G. Kutcher, S. Leibel, M. Zelefsky, T. LoSasso, S. Spirou, Q. Wu, J. Yang, J. Stein, R. Mohan, Z. Fuks, and C. C. Ling, "Planning, delivery, and quality assurance of intensity-modulated radiotherapy using dynamic multileaf collimator: A strategy for large-scale implementation for the treatment of carcinoma of the prostate," Int. J. Radiat. Oncol., Biol., Phys. 39, 863-873 (1997).

${ }^{12}$ G. A. Ezzell and S. Chungbin, "The overshoot phenomenon in stepand-shoot IMRT delivery," J. Appl. Clin. Med. Phys. 2, 138-148 (2001).

${ }^{13}$ D. L. Litzenberg, J. M. Moran, and B. A. Fraass, "Verification of dy- namic and segmental IMRT delivery by dynamic log file analysis," J. Appl. Clin. Med. Phys. (in press).

${ }^{14}$ J. M. Moran, B. A. Fraass, K. L. Lam, and D. L. Litzenberg, "An integrated system for DMLC (IMRT) fluence verification," Med. Phys. 28, 1284 (2001) (abstract).

${ }^{15}$ L. A. Dawson, K. K. Brock, S. Kazanjian, D. Fitch, C. J. McGinn, T. S. Lawrence, R. K. Ten Haken, and J. Balter, "The reproducibility of organ position using active breathing control $(\mathrm{ABC})$ during liver radiotherapy," submitted to Int. J. Radiat. Oncol. Biol. Phys. 51, 1410-1421 (2001). 